\title{
Between COVID-19 severity and its prevention - what should rheumatologists be aware of?
}

\author{
Joanna Makowska ID, Filip Styrzyński ID \\ Department of Rheumatology, Medical University of Lodz, Poland
}

A year has passed since the unprecedented eruption of SARS-CoV-2 infections affected almost all countries around the world. Rheumatic patients represent a numerous group of people in which the immune system is impaired both by chronic immunosuppressive treatment and by the disease itself. For this reason doctors are worried about the optimal management and how to prevent the exacerbation of autoimmune systemic diseases.

Recently, a few important studies have been published regarding the risk of a severe course of COVID amongst rheumatic patients, parallel to the dynamically ongoing worldwide vaccination process as never seen before.

Although the data remain scarce, Zhong et al. [1] suggested that patients with rheumatic musculoskeletal diseases (RMDs) might be almost 3 times more susceptible to COVID-19. Another study from France [2] assessed the risk of a severe course of COVID-19 in RMD patients. It showed that the risk of mortality amongst hospitalized individuals was almost 1.5 times higher than in the matched control group. This study also assessed risk factors for developing a severe disease - in multivariable analysis there were unfavourable known factors such as advanced age, male sex, obesity and hypertension as well as use of some antirheumatic drugs (glucocorticosteroids, rituximab and mycophenolate).

When considering the underlying rheumatic disorder, vasculitis, systemic autoimmune diseases and autoinflammatory diseases brought higher risk of a severe course compared to inflammatory arthritis. Nonetheless, use of methotrexate, tocilizumab and TNF inhibitors did not increase the risk of a fatal outcome. These findings shed some light on treatment choices in the COVID-19 era.

The study of Strangfeld [3] and participants of Global Rheumatology Alliance gathered data about the course of COVID-19 from patients with various rheumatic diseases coming from different countries and continents in. Factors associated with the fatal outcome of COVID-19 were advanced age, male gender, comorbidities (hypertension, cardiovascular disease, chronic lung disease) and moderate/high activity of the rheumatic disease. The use of higher doses of prednisolone (> $10 \mathrm{mg} /$ day), rituximab, sulfasalazine and some immunosuppressive drugs (azathioprine, cyclophosphamide, cyclosporine, mycophenolate and tacrolimus) was considered as an iatrogenic risk factor which increases the risk of death. Interestingly, the lack of DMARD treatment was associated with higher death risk compared to patients on methotrexate. Use of other biologicals or targeted synthetic DMARDs did not result in increased death risk. Also, in the study of Marques et al. [4] from Brazil, patients receiving high doses of immunosuppression (methylprednisolone or cyclophosphamide) had an increased risk of fatal outcomes, while treatment with TNF inhibitors seemed to have a protective role.

To sum up, the strongest risk factors for an unfavourable outcome are the same as in the general population, including advanced age, male sex and comorbidities such as diabetes, chronic lung disease or cardiovascular disease. Due to prolonged inflammation, autoimmune status and iatrogenic factors (e.g. chronic glucocorticosteroids treatment) patients with RMD and autoimmune systemic conditions are additionally prone to develop comorbidities associated with the elevated risk of a severe course of COVID-19 [5].

These findings support current guidelines of EULAR and other rheumatological societies, emphasizing the importance of tight control of the disease activity in the COVID-19 era. Unfortunately, according to research from Poland, problems with access to both primary and outpatient health care experienced by many patients resulted in worsening of the rheumatic disease in 2020. Despite initial enthusiasm towards the introduction of telemedicine [6], the rating of its usefulness dropped significantly after half a year of experience [article in press].

What seems very interesting is that the outcomes of COVID-19 treatments in RMD patients have changed over time. In the study of Jorge et al. [7] two cohorts of rheumatic patients infected with SARS-CoV-2 were com-

Address for correspondence:

Joanna Makowska, Department of Rheumatology, Medical University of Lodz, 113 S. Żeromski St., 90-549 Lodz, Poland, e-mail: joanna.makowska@umed.lodz.pl, ORCID: https://orcid.org/0000-0003-2036-375X

Submitted: 16.02.2021; Accepted: 19.02.2021 
pared. The early cohort (infections in the spring of 2020) had higher risk of hospitalization, mechanical ventilation, intensive care unit admission and fatal outcome compared to the late cohort (infections during summer months). These differences are multifactorial, including better testing and earlier diagnosis, the learning curve of medical staff, as well as the possible result of implementing remdesivir, dexamethasone or tocilizumab in the treatment strategies.

Since the outbreak of the pandemic, the race towards development of an effective and well-tolerated vaccine has started. Many rheumatological patients are eager to receive the COVID-19 vaccine, although the involvement of such individuals in the studies was rather incidental. Definitely none of the currently available or forthcoming vaccines in the EU should be even associated with a theoretical risk for infection amongst immunosuppressed patients, but their effectiveness has not yet been evaluated. The guidelines of rheumatological societies worldwide are mostly consistent, with some minor differences, such as rescheduling rituximab therapy. Nevertheless, all the authors agree on the favourable risk profile of the vaccinations. Some conclusions on immunogenicity might be therefore encouraged by the previous research on influenza and pneumococcal vaccines.

Subesinghe et al. [8] stated that the humoral response was preserved amongst methotrexate or TNFi patients to the influenza vaccine, reduced in methotrexate, but not the TNFi group towards 6B/23F pneumococcal serotype, and was limited in the combination of methotrexate/TNFi to both vaccines. On the other hand, Park et al. [9] found in a randomized controlled trial that 2-week discontinuation of methotrexate, following vaccination of patients with rheumatoid arthritis, improves the humoral response to the influenza vaccine without exacerbation of the disease activity. According to Hua et al. [10] rituximab reduced the immunological response to both the influenza and pneumococcal vaccine. Other popular DMARDs do not seem to have a major influence, although large prospective studies are needed.

Although initially possible increased risk for severe allergic reactions towards COVID-19 vaccines was considered, the current data from the US (as of January $18^{\text {th }}$ ) describe anaphylaxis as a rare event (4.7 and 2.5 cases/ million for Pfizer and Moderna respectively), without any fatal cases reported [11]. According to an EAACI statement, there is no contraindication to administer COVID-19 vaccines under appropriate conditions apart from a history of an allergic reaction to any of the product components [12].

In the light of recently published data on probable higher risk of a severe course of COVID-19 infection in rheumatic patients, vaccination should be widely recommended, as up to now this seems to be a safe and effective way to prevent health decline.

The authors declare no conflict of interest.

\section{References}

1. Zhong J, Shen G, Yang H, et al. COVID-19 in patients with rheumatic disease in Hubei province, China: a multicentre retrospective observational study. Lancet Rheumatol 2020; 2: E557-E564, DOI: 10.1016/S2665-9913(20)30227-7.

2. FAI2R/SFR/SNFMI/SOFREMIP/CRI/IMIDIATE consortium and contributors. Severity of COVID-19 and survival in patients with rheumatic and inflammatory diseases: data from the French RMD COVID-19 cohort of 694 patients. Ann Rheum Dis 2020; annrheumdis-2020-218310, DOI: 10.1136/annrheumdis2020-218310 [Online ahead of print].

3. Strangfeld $A$, Schäfer $M$, Gianfrancesco $M A$, et al. Factors associated with COVID-19-related death in people with rheumatic diseases: results from the COVID-19 Global Rheumatology Alliance physician-reported registry. Ann Rheum Dis 2021; annrheumdis-2020-219498, DOI: 10.1136/annrheumdis2020-219498 [Online ahead of print].

4. Marques CDL, Kakehasi AM, Pinheiro MM, et al. High levels of immunosuppression are related to unfavourable outcomes in hospitalised patients with rheumatic diseases and COVID-19: first results of ReumaCoV Brasil registry. RMD Open 2021; 7: e001461, DOI: 10.1136/rmdopen-2020-001461.

5. Fitzgerald GE, Maguire S, Haroon N. COVID-19: What Do Rheumatologists Need to Know? Curr Rheumatol Rep 2021; 23: 5, DOI: 10.1007/s11926-020-00971-y.

6. Opinc A, Łukasik Z, Makowska J. The attitude of Polish rheumatology patients towards telemedicine in the age of the COVID-19 pandemic. Reumatologia 2020; 58:134-141, DOI: 10.5114/reum.2020.96665.

7. Jorge A, D'Silva KM, Cohen A, et al. Temporal trends in severe COVID-19 outcomes in patients with rheumatic disease: a cohort study. Lancet Rheumatol 2021; 3: E131-E137, DOI: DOI:10.1016/S2665-9913(20)30422-7.

8. Subesinghe S, Bechman K, Rutherford Al, et al. A Systematic Review and Metaanalysis of Antirheumatic Drugs and Vaccine Immunogenicity in Rheumatoid Arthritis. J Rheumatol 2018; 45: 733-744, DOI: 10.3899/jrheum.170710.

9. Park JK, Lee YJ, Shin K, et al. Impact of temporary methotrexate discontinuation for 2 weeks on immunogenicity of seasonal influenza vaccination in patients with rheumatoid arthritis: a randomised clinical trial. Ann Rheum Dis 2018; 77: 898-904, DOI: 10.1136/annrheumdis-2018-213222.

10. Hua C, Barnetche T, Combe B, Morel J. Effect of methotrexate, anti-tumor necrosis factor $\alpha$, and rituximab on the immune response to influenza and pneumococcal vaccines in patients with rheumatoid arthritis: a systematic review and metaanalysis. Arthritis Care Res (Hoboken) 2014; 66: 1016-1026, DOI: 10.1002/acr.22246.

11. Shimabukuro TT, Cole M, Su JR. Reports of Anaphylaxis After Receipt of mRNA COVID-19 Vaccines in the US - December 14, 2020-January 18, 2021. JAMA 2021, DOI: 10.1001/ jama.2021.1967 [Online ahead of print].

12. Sokolowska M, Eiwegger T, Ollert M, et al. EAACl statement on the diagnosis, management and prevention of severe allergic reactions to COVID-19 vaccines. Allergy 2021, DOI: 10.1111/ all.14739 [Online ahead of print]. 